\begin{tabular}{lllll}
\hline Estudios de Economía Aplicada & Vol. 36-1 & 2018 & PÁgs. 21-34 \\
\hline
\end{tabular}

\title{
Hacia una nueva Economía Aplicada
}

\section{Antonio Pulido San Román}

Instituto L.R. Klein (Centro Stone). Universidad Autónoma de Madrid, c/ Francisco Tomás y Valiente , 5, 28049 Madrid, España.E-mail: antonio.pulido@uam.es

\section{RESUMEN}

Revisión de la separación de la economía en teórica y aplicada. Tendencias en la evolución del contenido de los textos universitarios de Economía. Los rasgos de futuro de una nueva economía aplicada y su conexión con el mundo profesional.

Palabras clave: Economía aplicada, economía académica, profesiones, innovación.

\section{Towards a New Applied Economics}

\begin{abstract}
Review of the separation of the economy into theoretical and applied. Trends in the evolution of the content of university economics texts. The future characteristics of a new applied economy and its connection with the professional world.
\end{abstract}

Keywords: Applied Economics, Academic Economics, Professions, Innovation.

Clasificación JEL: B40

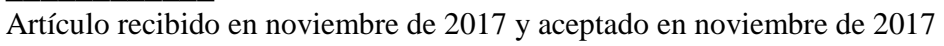

Artículo disponible en versión electrónica en la página www.revista-eea.net, ref. ə-36107 


\section{HACIA UNA NUEVA ECONOMÍA RENOVADA.}

Aplicar la ciencia económica no es una originalidad de nuestro campo de conocimiento. También se utiliza el término "aplicada" (con carácter internacional) a la antropología, física, lingüística, biología, psicología cognitiva o incluso a la teología.

Según el Cambridge Dictionary la aplicación en economía supone "un estudio en la relación con situaciones reales, más allá de la teoría económica”. El Journal of Applied Economics publica aplicaciones en micro y macroeconomía, a problemas en los sectores públicos o privados que ayudan a acercar la teoría económica a la realidad.

Por tanto, una Nueva Economía Aplicada (NEA) debe establecerse a partir de una ciencia económica renovada, aunque tenga otros múltiples aspectos a evaluar a los que nos referimos anteriormente.

Cuando me refiero a una Nueva Economía Aplicada incluyo los cambios que ya han podido percibirse en los últimos años, así como las tendencias previsibles en un futuro a corto y medio plazo. Se refieren a los cambios disruptivos de la sociedad del conocimiento y más específicamente de la economía digital, pero también a los nuevos rumbos de una economía más implicada en cuestiones sociales y con mayor componente cualitativo e interdisciplinar.

Sin embargo, es muy complejo el debate sobre el estado de aceptación actual, razones de cambio y contenido de esa economía renovada. Se mezclan posiciones metodológicas dispares, escuelas de pensamiento, ideologías políticas diferentes e, incluso, unos límites imprecisos de las fronteras de la economía y la dicotomía teórico/aplicada.

A mi entender, la división entre economía "teórica” y "aplicada” es de una debilidad conceptual extrema y solo se explica por matices sobre su contenido más o menos empírico, organización docente de las universidades y recelos/luchas de poder entre departamentos y/o profesores.

La economía es, en su esencia, un campo del saber aplicado y cuyo contenido empírico (datos) y cercanía a los problemas reales es indiscutible. Como todo conocimiento científico, hay un campo la investigación básica, no guiada por la aplicabilidad inmediata. Pero es un reducto limitado para investigadores teóricos, con una producción de uso interno en las universidades o centros de investigación.

Personalmente siempre he defendido la importancia de la investigación básica en todos los campos. Muchos avances en investigación aplicada y desarrollo tecnológico y de nuevos productos tienen su origen en avances teóricos previos.

Sin embargo, la teoría pura en campos científicos muy orientados a la acción, como la economía y la administración de empresas, no creo que deba vivir aislada de la experimentación y contraste empírico de hipótesis y teorías. 
Tras una amplia experiencia en la utilización de modelos econométricos (punto de unión entre modelos teóricos y técnicas matemático-estadísticas) a escala macro, meso y micro, me inclino por una economía integradora de teoría y aplicación.

Las teorías puras, macro o micro, no proporcionan leyes válidas universales. Responden a hipótesis, muchas veces restrictivas, ideales, que pueden no ser válidas para describir y analizar la situación de muchas realidades concretas.

Pero incluso con el filtro de técnicas econométrico-estadísticas no es posible obtener leyes empíricas de validez amplia y permanente. Las realidades socioeconómicas de países o empresas cambian continuamente en un mundo en profunda transformación tecnológica, demográfica, sectorial o empresarial.

En mi opinión (y la de un número creciente de académicos) la economía debe acercarse más a la realidad que trata de descubrir y analizar. Más que dos versiones de economía, una teórica y otra aplicada, debemos buscar y difundir una visión integradora.

A mediados del siglo XIX, John Stuart Mill publicó un texto que fue referencia durante medio siglo: Principles of Political Economy. A principios del siglo XX le sucedía como "core” de conocimiento académico los Principles of Economics de Alfred Marshall. En 1936 John Maynard Keynes publica General Theory of Employment, Interest and Money, con un contenido sensiblemente más empírico.

Como estudiante, personalmente viví la profunda transformación de la enseñanza de la economía que respondía a las renovadas teorías keynesianas y a la gran síntesis neoclásica del texto líder de Paul Samuelson, Curso de Economía Moderna, cuya edición inicial se realizó en 1948.

Era un libro de teoría pero lleno de referencias a la realidad económica, de cifras, que ayudaban a concretar ideas aplicables a los países analizados. En su edición española de 1958 subrayaba su "fobia particular hacia los libros cuyas cifras están ya anticuadas de algunos años a la publicación de aquellos...Solo lamento no poder acercarlo más a 1960"

Desde entonces cambian las teorías, se amplían los temas estudiados, se acerca el análisis de leyes generales a la realidad. Pero demos un paso más y podemos referirnos al nuevo texto online The Economy (2017), elaborado bajo el patrocinio del "think-tank" británico New Economic Fundations, por un amplio grupo de más de 20 profesores (The CORE Team).

Creo que puede resultar útil repasar el nuevo contenido actualizado de una economía que cambia como lo hace el mundo al que debe aplicarse sus conocimientos. Incluyo en la Tabla adjunta (Tabla 1) el título de los 22 capítulos del curso. 
Tabla 1

Capítulos del curso

\begin{tabular}{|ll|}
\hline 1. & La revolución capitalista \\
2. & Tecnología, población y crecimiento \\
3. & Escasez, trabajo y elección \\
5. & Interacciones sociales \\
6. & La empresa: Propietarios, directivos y empleados \\
7. & La empresa y sus consumidores \\
8. & Oferta y demanda: Tomadores de precio, mercados competitivos \\
9. & El mercado del trabajo: salarios, beneficios y desempleo \\
10. & Bancos, dinero y mercado de crédito. \\
11. & Dinámica de mercado, búsqueda de rentas y establecimientos de precios \\
12. & Mercados, eficiencia y política pública \\
13. & Fluctuaciones económicas y desempleo \\
14. & Desempleo y política fiscal \\
15. & Inflación, desempleo y política monetaria \\
16. & Progreso tecnológico, empleo y estándar de vida a largo plazo \\
17. & Revisión final: La Gran Depresión, la época dorada y la crisis financiera \\
18. & Revisión final: La nación y la economía global \\
19. & Revisión final: Economía de la desigualdad \\
20. & Revisión final: Economía y medioambiente \\
21. & Revisión final: Innovación, información y la economía de redes \\
22. & Revisión final: Economía, política y política pública \\
\hline
\end{tabular}

Fuente: The CORE Team. The Economy (2017).

Estamos refiriéndonos a una economía renovada junto a una nueva forma de aprender economía (Cassidy, 2017). La competencia perfecta no es la norma sino un caso particular de la competencia; debe analizarse la desigualdad y el reparto de las ganancias generadas por el comercio, la innovación o las finanzas; el PIB es una medida de crecimiento económico con fallos para medir el progreso; atención especial a la innovación industrial y la economía de la información...

Los aires de cambio de la economía soplan desde muchos rincones. Por citar algunos de los más recientes:

- Institute for New Economic Thinking (www.ineteconomics.org). "Nuevas ideas económicas para mejor servicio a la humanidad” Reunión Edimburgo, Octubre 2017, sobre el despertar de una nueva economía adaptada a los cambios de nuestro tiempo.

- Schumacher Center for New Economics (www.centerforneweconomics.org) "Combinación de investigación teórica en economía con aplicación práctica enfocada a transformar sistemas hacia una mayor sostenibilidad para personas y el planeta”. 
- Institute for New Economic Thinking at the Oxford Martin School (www.inet.ox.ac.uk) "El mundo necesita un modelo de crecimiento económico y prosperidad que sea más inclusivo, sostenible y robusto".

- Evonomics, The Next Evolution of Economics (www.evonomics.com) "New Economic foundation for de new economy".

Entre los libros recientes que inciden en los cambios a seguir por esta nueva economía, citaré el de Richard Bookstaber (2017) sobre el fin de la teoría como consecuencia de la crisis financiera, los fallos de la economía y el barrido de la interacción humana. Bookstaber no es un profesor universitario, sino un veterano en la gestión de riesgos financieros y, por tanto, su visión puede valorarse como parcial aunque interesante: la prueba de la incapacidad de la teoría económica convencional está en su proceso para detectar, evaluar y corregir la pasada crisis financiera.

Mi posición personal la he resumido en varios post de mi blog de los últimos tres años. En el titulado ¿Críticas a la economía académica? (27/09/2015) comento que la economía que se investiga y enseña desde las universidades ha sido criticada desde distintos puntos de vista. No es un fenómeno reciente pero sí de plena actualidad, con variantes propias por países, centros e incluso materias. Se ha argumentado, en general, su distanciamiento del mundo real, su uniformidad ideológica, la preocupación de los profesores por lo que repercute directamente en su carrera académica,...

La "torre de marfil” en que se encierran algunos profesores de Economía, me parece un error imperdonable en cualquier caso y, mucho más, en el momento actual en que los temas económicos y empresariales son de interés y difusión social generalizada.

Pero hay, también, que reconocer que la realidad como objetivo último de análisis tiene un límite, especialmente en las áreas de conocimiento cercanas a la investigación básica. La Universidad debe mantener un equilibrio entre la aplicabilidad a corto plazo de los conocimientos que desarrolla y los cimientos científicos de su planteamiento a largo. La Universidad no puede ni debe ser, ni una academia de aprendizaje, ni una consultora para aplicaciones y servicios de apoyo rutinario a la Administración Pública o a las empresas. La investigación exige innovación de conocimientos y búsqueda de caminos inexplorados que pueden no dar resultados de momento. Lo importante es mantener un adecuado equilibrio.

El problema se plantea cuando una parte importante de la $\mathrm{I}+\mathrm{D}+\mathrm{i}$ que se genera en esa gran Academia del Pensamiento que es la Universidad, sigue objetivos que tienen más que ver con intereses propios de los profesores que con un proyecto de mejora del conocimiento que pueda, razonablemente, terminar siendo útil a la sociedad. 
De aquí nace toda una corriente crítica con una "economía académica" alejada de situaciones que puedan darse en la realidad y principalmente preocupada por la estética y el prestigio de desarrollos teóricos complejos, con una habitual alta carga matemática-estadístico-econométrica.

Aunque hay múltiples manifestaciones en contra de esa deriva de la investigación económica, algunos excesos han puesto el tema de plena actividad. A título de ejemplo citaré la de un prestigioso profesor de la New York University, Paul Romer (2015), que en un congreso de la American Economic Association (2015) presentó una ponencia en que denunciaba la "matematicidad" o "matematicismo" extremos ("mathiness" es el término empleado) en la teoría del crecimiento económico. Más allá de la polémica a que dio lugar entre los especialistas en crecimiento, lo que parece más relevante es la denuncia de que se manejan hipótesis irrealistas (p.ej. competencia perfecta en los mercados) detrás de construcciones matemáticas complejas.

Es cierto, además, que se ha producido una tendencia a confundir matematización con rigor y calidad científica en una ciencia social como la economía (y la administración de empresas). Mi experiencia personal de más de cinco años como presidente de la Comisión de la ANECA evaluadora del profesorado universitario en el amplio campo de las ciencias sociales, me confirma en esta confusión. Exigir calidad en la producción científica es un requisito imprescindible. Considerar que en el amplio campo de la $\mathrm{I}+\mathrm{D}+\mathrm{i}$, la calidad sólo existe en artículos publicados en un número reducido de revistas internacionales de prestigio es una simplificación excesiva que desconoce la posible arbitrariedad de los jueces y los círculos de poder académicos. Partir de la premisa de que la calidad en economía exige matematización y planteamientos complejos e inéditos, puede conducir a irrealismos que sitúan la estética del mensaje por encima de su utilidad ¡En cuántos artículos o tesis doctorales prima la especificación de un modelo o técnica de cálculo extremadamente compleja sobre el conseguir un avance en el campo de conocimiento seleccionado, incluso dentro del área de la economía aplicada!

Como profesor de Econometría sería difícil creer (y por supuesto erróneo) que estoy en una posición opuesta al uso de modelos, a la cuantificación de los fenómenos económicos o a la utilidad de la prospectiva o la predicción económica y empresarial. Pero los excesos y defectos siempre es necesario ponerlos de manifiesto.

Ya en la sutil frontera entre economía teórica y aplicada, en un post sobre los conocimientos de los economistas (11/09/2017) matizaba mi opinión personal en siete puntos:

- Aunque tardé años en admitirlo, debo reconocer que la Economía no es ajena a planteamientos políticos, morales y sociales. No es una ciencia 
con proposiciones plenamente objetivas, sino condicionadas por creencias y un amplio y variado contexto que la rodea.

- Por tanto no hay verdades absolutas, sino acercamientos teóricos y prácticos en general discutibles. En particular es dañino para la sociedad no reconocer condicionantes ideológicos y resulta incluso fraudulento buscar resultados parciales que se presenten como garantía de objetividad en las propuestas.

- Las fronteras de la Economía son muy amplias y deben incluir aspectos institucionales, geopolíticos, medioambientales, tecnológicos,...Cerrar fronteras es un suicidio científico y profesional.

- La interdisciplinariedad debe ser una exigencia metodológica y de práctica profesional del economista. La Economía trata de comportamientos de individuos y colectivos; exige planteamientos psicológicos, sociológicos y humanísticos en su más amplio espectro.

- La información estadística disponible condiciona los análisis de los economistas. Las limitaciones en cuestiones de interés público deben corregirse.

- Todos debiéramos reconocer que la Economía está al servicio de la sociedad y no es, por tanto, ni un asunto exclusivo de economistas, ni priman los objetivos estrictamente económicos.

- En resumen: La crítica a los economistas no debiera centrarse en lo que sabemos, sino en cómo aplicamos nuestros conocimientos en un contexto cada día más complejo y en profundo cambio, en el que compartimos terreno de juego con otros múltiples profesionales.

\section{SOBRE LA RENOVACIÓN A FUTURO DE LA ECONOMÍA APLICADA Y LA PROFESIÓN DE ECONOMISTA.}

Hasta ahora me he referido a la economía como un área de conocimiento global, principalmente centrada en temas macro. Pero si pasamos del mundo académico al profesional y aceptamos, como referencia simplista pero cierta utilidad práctica, que economía es lo que hacen los economistas, los conocimientos que predominan ampliamente son los aplicados sobre los teóricos.

En el libro blanco del Título de Grado en Economía y Empresa, la ANECA (2015) detalla algunas salidas profesionales y competencias generales:

- Servicios de estudio y planificación

- Fiscalidad

- Administración Pública

- Organismos internacionales 
- Comercio Exterior

- Dirección o Gerencia de Empresas

- Consultoría económica

- Docencia e investigación

- Competencia en herramientas digitales

- Competencia en recoger, tratar y analizar la información sobre situación y evolución de una organización o de la economía en general

En los últimos años los grados que se cursan en las universidades españolas han evolucionado desde Economía y ADE a más de veinte, que marcan especializaciones nuevas en un proceso constante de adaptación a las necesidades formativas. Aparte de un creciente número de títulos mixtos, unos pocos ejemplos:

- Consultoría y gestión de la Información

- Gestión de Pequeñas y Medianas empresas

- Comercio

- Economía y Finanzas

- Finanzas y Contabilidad

- Economía y Negocios Internacionales

- Marketing

- Fiscalidad y Administración Pública

- Liderazgo Emprendedor e Innovador

- Administración y Dirección de empresas tecnológicas

- ADE y Gestión de la innovación

No es cuestión de entrar en el detalle de las profesiones en que pueden estar implicados los economistas (en sentido amplio) pero, a efectos de la Nueva Economía Aplicada (NEA) hay que partir de su diversidad creciente y la no equivalencia estricta entre formación académica y actividad profesional.

En un post sobre esta variada y dinámica profesionalización de los economistas (19/09/17), señalo que en muchos campos profesión y titulación son casi inseparables. Son marginales y escasos los casos de "espontáneos", "aficionados" o "intrusos" en medicina o ingeniería. Pero no ocurre lo mismo con profesiones vinculadas a conocimientos de aplicación múltiple, como filología, matemáticas, historia, comunicación...o gestión económica y empresarial.

Excepto en campos muy especializados reservados a titulados en Ciencias Económicas y Empresariales, los profesionales dedicados a dirección de empresas, gestión financiera o de ventas, tratamiento de la información, 
“coaching”, turismo, mercados internacionales, consultoría, publicidad o medio ambiente, provienen de formaciones muy diversas: economistas, ADE, psicólogos, ingenieros, informáticos,...

Más aún: en un mundo tan cambiante y polivalente como el actual, escasean cada día más los puestos reservados para un perfil definido de profesionales. En una "formación de por vida" el sello del título de formación superior no garantiza un destino profesional fijo. Para el conjunto de las profesiones debe partirse del hecho de que menos de una tercera parte consigue trabajar en lo que estudia. Por tanto, no estudiamos para un trabajo, sino que debemos atender a una formación en competencias y capacidades que facilitan nuestra empleabilidad de por vida en un mundo rápidamente cambiante.

Así en el Foro de Economistas del Consejo General de Economistas correspondiente a 2016, se trataron temas diversos como "La formación universitaria de los economistas en España” (Ana López, 2016)

Se destacó la necesaria conexión entre formación y una empleabilidad que exige, cada día más:

- Perfil multidisciplinar

- Gran polivalencia

- Capacidad analítica y orientación a resultados

- Visión estratégica

- Proactividad y adaptación al cambio

Por supuesto, el desafío no es exclusivo de la formación económica, sino que afecta a las habilidades que parece van a pedirse en el mundo del trabajo. Según World Economic Forum (Alex Gray, "The 10 skills you need to thrive in the Fourth Industrial Revolution"), estas habilidades para un horizonte tan cercano como 2020 serían, con éste orden de prioridad:

- Resolución de problemas complejos

- Pensamiento crítico

- Creatividad

- Gestión de equipos

- Coordinación con otras personas

- Inteligencia emocional

- Criterio y toma de decisiones

- Orientación al servicio

- Negociación

- Flexibilidad cognitiva 
Pero lo más relevante, también en mi opinión, es el recordatorio de que la mayoría de los economistas son fontaneros, en la aceptación ya utilizada por Esther Duflo (2017): "aquellos que tienen una serie de herramientas que aplicadas metódicamente resuelven el 99\% de los problemas y cuestiones económicas de la vida diaria”. Estos son los frecuentemente olvidados gestores o asesores, a escala macro o de empresa u otras instituciones, en temas financieros, comerciales, contables, fiscales,...

Los posibles errores y aciertos de los economistas (y de los que actúan en funciones, tengan o no título oficial) deben analizarse referidos a los diferentes grupos profesionales. Como académicos, podemos debatir sobre el realismo y capacidad explicativa de teorías y modelos económicos. Como economistas aplicados, las recetas exigen una mayor cercanía al diverso y cambiante mundo profesional.

La NEA debe prestar una atención especial a las tecnologías de mayor impacto futuro tales como:

- Internet de las Cosas

- Realidad virtual y aumentada

- Inteligencia artificial

- Impresión 3D

- Drones

- Robots

- Blockchain

En el terreno de la gestión será prioritario atender a los múltiples riesgos con que los economistas aplicados se van encontrando: riesgos tecnológicos, financieros, geopolíticos, operativos, estratégicos o de mercado.

En el propio campo profesional los economistas aplicados deberán enfrentarse a nuevas tendencias: oficinas online, globalización, competencia de nuevas profesiones, logística internacional, cambios demográficos, aprendizaje de por vida,...

En cuanto a la estrategia empresarial, están cambiando los patrones más habituales. Aparecen nuevas empresas rupturistas (unicornios, exponenciales, cisnes negros) y se tiende a una estrategia más disruptiva que anticipe los retos de futuro y facilite los cambios organizativos provocados por mercados de expansión, desbloqueo de activos, cadenas de valor, plataformas, etc.

La NEA exige organizaciones más flexibles e innovadoras que aprovechan oportunidades de las nuevas tecnologías TIC en una renovada y pujante sociedad de la información y el conocimiento. 
Vislumbrar las claves de futuro y utilizar adecuadamente las técnicas de predicción económica y empresarial, jugarán un papel creciente en esa NEA. La predicción va acortando plazos y acercándose cada día más a la toma de decisiones. Los datos utilizados han ido pasando de anuales a trimestrales, mensuales, semanales, diarios u horarios. Las herramientas de predicción, a partir de esos datos, han ido acortando tiempos de respuesta y la evolución es imparable.

Pero tener muchos datos, actualizarlos permanentemente y disponer de programas informáticos cada vez más inteligentes y autónomos, no es garantía de una predicción útil para tomar decisiones acertadas. La información no siempre se transforma en conocimiento. El automatismo y la celeridad en buscar resultados pueden llevar a proyecciones de futuro ingenuas. El futuro es muy complejo y no se debe caer en la idolatría de unas cifras de predicción cambiantes con las expectativas de las personas o las acciones de las empresas competidoras en un entorno global. Y menos aún en carecer de apuestas de futuro.

El Institute for the Future, un centro non-profit norteamericano especializado en predicción a largo plazo y métodos de investigación de futuros, publicó algunos datos interesantes en su informe "The Information Generation. Transforming the future, today" (2015)

En una encuesta realizada a 3.600 líderes empresariales de 18 grandes economías del mundo, se citaba cómo la implicación de futuro más relevante de las tendencias en la generación y tratamiento de datos, era potenciar la predicción de nuevas oportunidades de negocio". Un 60\% de los ejecutivos empresariales la citan como la más relevante (en China llegaba al 71\%), seguida por "innovar de una forma más ágil” y "mostrar transparencia y credibilidad". Sin embargo, reconocían los directivos que sólo 1 de cada 3 utilizaba efectivamente esa nueva capacidad para adelantarse al futuro.

En un entorno predominante cortoplacista en la gestión económica pública y privada, es necesario apuntar la necesidad de planteamientos estratégicos. Las piernas pueden ejercitarse para recorrer espacios de gestión al nanosegundo; pero la cabeza de las organizaciones debe reflexionar, con calma, en términos de futuro. Esos deben ser también rasgos característicos de la NEA.

Una última llamada de atención en la evolución hacia esa NEA. Sí la macroeconomía ya hemos visto que va evolucionando hacia una incorporación más amplia de aspectos sociales, psicológicos, geopolíticos y tecnológicos, esas tendencias no deben ser ajenas a la economía aplicada empresarial.

Un centro dedicado a estrategia empresarial (BGC Henderson Institute, 2017) insiste en que la empresa ya no debe estar solo atenta al mundo de los negocios y su estrategia competitiva, innovación, operaciones y recursos humanos. Ese era el juego de ayer y resultaba relativamente fácil de jugar. Ahora 
los lideres (economistas o no) deben jugar en el terreno desconocido de altos niveles de incertidumbre e inestabilidad, en una economía global con nuevas reglas. Su mentalidad debe acercarse más a las "matrioskas" rusas que al ajedrez. Las empresas son una parte anidada en sistema adaptativos complejos, cuyos niveles más altos corresponden a la economía internacional, nacional, local y sectorial.

Pero, además, las empresas del futuro deben también considerar aspectos sociales más allá del puro negocio, con una visión a largo plazo. Como señala Laula Walid, directora de la División de Finanzas del grupo Bosch en Stuttgart, el "controlling” en la gestión empresarial debe estar vinculado a asegurar la supervivencia de la empresa a largo plazo, compartiendo responsabilidades sociales de generación y de mantenimiento de empleos y estabilidad del conjunto de la economía.

En resumen, creo que en esa Nueva Economía Aplicada, teoría, datos, modelos y técnicas de análisis deberán integrarse con planteamientos psicológicos, sociológicos, tecnológicos o geopolíticos en unos entornos VICA (volátiles, inciertos, complejos y ambiguos) Pero, además, se acercarán a una macroeconomía más cercana a la realidad y preocupada por analizar los problemas de la sociedad, con una gestión empresarial más pendiente del entorno.

\section{REFERENCIAS BIBLIOGRÁFICAS}

ANECA (2015). Libro Blanco. Título de Grado en Economía y en Empresa

BOOKSTABER, R. (2017). The end of theory. Princeton University Press.

BOWLES, S.; CARLIN, W. Y STEVENS, M (coordinadores del CORE Team) (2017).

"The economy", www.core-econ.org/the-economyl

CASSIDY, J. (2017). "A new way to learn economics". The New Yorker, (11/09/17).

DUFLO, E. (2017). "The Economist as Plumber". ASSA meeting.

GRAY, A. (2016). "The 10 skill you need to trive in the Fourth Industrial Revolution". World Economic Forum (post 19/01/16).

INSTITUTE FOR THE FUTURE (2015). "The information generation: Transforming the future today". EMC.

LÓPEZ, A. (2016). "La formación universitaria de los economistas en España: retos en

el Espacio Europeo de Educación Superior". Consejo General de Economistas.

PULIDO, A. (2017). “¿Qué saben los economistas?”. Blog Antonio Pulido (11/09/17).

PULIDO, A. (2017). "La variada tribu de los economistas". Blog Antonio Pulido $(19 / 09 / 17)$.

PULIDO, A. (2015). “¿Críticas a la economía aplicada?”. Blog Antonio Pulido (27/09/15). 
REEVES, M. Y HARNOSS, J. (2017). "The business of business is no longer just business". BCG Henderson Institute.

ROMER, P. (2015). "Mathiness in the theory of economic growth". AEA Papers and Proceedings.

WALID, L. (2017). "El controlling en la gestión empresarial". Instituto de Dirección y Organización de Empresas, Universidad de Alcalá, Documento na 82, abril 2017. 
